\title{
Knotted Solitons in Nonlinear Magnetic Metamaterials
}

\author{
Nikolay N. Rosanov, ${ }^{1,2}$ Nina V. Vysotina, ${ }^{2}$ Anatoly N. Shatsev, ${ }^{2}$ Anton S. Desyatnikov, ${ }^{3}$ and Yuri S. Kivshar ${ }^{1,3}$ \\ ${ }^{1}$ National Research University of Information Technologies, Mechanics, and Optics (ITMO), St. Petersburg 197101, Russia \\ ${ }^{2}$ Vavilov State Optical Institute, St. Petersburg 199034, Russia \\ ${ }^{3}$ Nonlinear Physics Centre, Research School of Physics and Engineering, The Australian National University, \\ Canberra ACT 0200, Australia
}

(Received 21 December 2011; published 30 March 2012)

\begin{abstract}
We demonstrate that nonlinear magnetic metamaterials comprised of a lattice of weakly coupled splitring resonators driven by an external electromagnetic field may support entirely new classes of spatially localized modes-knotted solitons, which are stable self-localized dissipative structures in the form of closed knotted chains. We demonstrate different topological types of stable knots for the subcritical coupling between resonators and instability-induced breaking of the chains for the supercritical coupling.

DOI: 10.1103/PhysRevLett.108.133902

PACS numbers: 42.65.Tg, 42.65.Hw, 42.65.Pc
\end{abstract}

Knots appear in different branches of science, from knotted molecules in biology [1] and chemistry [2] and knot invariants in statistical mechanics [3] to liquid crystal defects [4] and vortex tangles in excitable media [5]. It is the idea of vortex knots in ether by Lord Kelvin [6] that stimulated the early development of knot theory in the late 19 th century. Vortices in electromagnetic fields are quantized phase defects with zero intensity, dark spots in two dimensions, and "threads of darkness" in the bulk, and optical vortex knots were predicted theoretically [7] and observed in experiments with laser beams [8].

In nonlinear fields, knotted solitons were introduced by Faddeev and Niemi [9] as knotted lines without selfcrossings embedded into a three-dimensional space. The existence of knot solitons as stationary solutions of nonlinear models was confirmed in numerical experiments [10]. Knotted solitons were also predicted for the models of spinor Bose-Einstein condensates [11] and multicomponent superconductors [12].

In this Letter, we predict that nonlinear magnetic metamaterials composed of lattices of resonant elements such as split-ring resonators (SRRs) can support a variety of localized dissipative patterns, including stable dissipative knotted solitons. Solitons in dissipative systems are, in general, far more robust than conservative solitons [13], and thus they are excellent candidates for the first realization of stable knotted solitons in the experiment.

A suitable model that supports stable localized modes such as knotted lines should be discretized with the possibility to tune the dissipation and gain at each site (or "meta-atom") as well as the interatomic coupling. We consider a nonlinear magnetic metamaterial comprised of a cubic lattice of weakly coupled SRRs [14-18]. Such a system exhibits a wealth of nonlinear dissipative phenomena, including bistability, modulational instability, switching waves, and one-dimensional dissipative solitons [15]. Taking this model as an example of a general discrete dissipative system, we analyze, for the first time to our knowledge, knotted dissipative solitons in such discrete systems and study their stability.

We begin with the equation for slowly varying amplitudes of the electric currents $\psi_{\mathbf{n}}$ in co-oriented SRRs at the discrete locations in cubic lattice, $\mathbf{n}=\left\{n_{x}, n_{y}, n_{z}\right\}$, with integer $n_{j}=1 \ldots N_{j}$; here, $j=x, y, z$. In the dimensionless form, it can be derived as [15]

$$
i \frac{d}{d t} \psi_{\mathbf{n}}-\left(2 \Omega-i \gamma+\alpha\left|\psi_{\mathbf{n}}\right|^{2}\right) \psi_{\mathbf{n}}-\Sigma=k C_{\mathbf{n}} .
$$

Here, $t$ is the time normalized to the period of the eigenmodes of a single SRR and $\Omega$ and $\gamma$ are both normalized to the eigenfrequency of a single SRR and denote, respectively, the detuning of the eigenfrequency from the frequency of the external pump radiation with amplitude $\Sigma$ and the current damping coefficient. Coefficient $\alpha=\mp 1$ for (de-)focusing cubic nonlinearity. The coefficient $k$ on the right-hand side defines the strength of the anisotropic magnetoinductive coupling between nearest neighbors,

$$
\begin{aligned}
C_{\mathbf{n}}= & 2\left[\psi\left(n_{x}, n_{y}, n_{z}-1\right]+\psi\left[n_{x}, n_{y}, n_{z}+1\right)\right] \\
& -\left[\psi\left(n_{x}-1, n_{y}, n_{z}\right)+\psi\left(n_{x}+1, n_{y}, n_{z}\right)\right] \\
& -\left[\psi\left(n_{x}, n_{y}-1, n_{z}\right)+\psi\left(n_{x}, n_{y}+1, n_{z}\right)\right] .
\end{aligned}
$$

Here, we denote $\psi_{\mathbf{n}}=\psi\left(n_{x}, n_{y}, n_{z}\right)$. Importantly, as discussed in detail in Refs. $[19,20]$, the anisotropy as well as the relative strengths of the coupling $k_{j}$ in different directions (here, $k=k_{x}=k_{y}=-k_{z} / 2$ ) are determined by the mutual orientation of SRRs. Therefore, varying the resonator's orientation within the lattice as well as building lattices of different symmetries (triangular, etc.) allow for more complex generalizations of this system. Equation (1) assumes uniformly distributed pump illumination $\Sigma$, the approximation which might fail in deep layers because of the radiation screening by the lattice. However, for small number of layers and weak coupling between resonators, this effect can be neglected; it can be also avoided by using an independent pump for each layer. 
For small coupling $k$, the perturbative approach is effective, starting from the anticontinuum limit in Eq. (1) with vanishing coupling $k \rightarrow 0$ and fully independent oscillators. Note also that the coupling term in Eq. (2) vanishes for solutions with homogeneous magnetization, $\psi_{\mathbf{n}}(t)=$ $\psi(t)$, i.e., $C_{\mathbf{n}}[\psi(t)] \equiv 0$. In both these cases [Eq. (1) with a zero right-hand part], the stationary solutions, $\psi(t)=\phi=$ const, satisfy $|\phi|^{2}\left[\gamma^{2}+\left(2 \Omega+\alpha|\phi|^{2}\right)^{2}\right]=|\Sigma|^{2}$, independent of coupling $k$. The bistable regime [15], with two stable states $\left|\phi_{\downarrow}\right|<\left|\phi_{\uparrow}\right|$, is realized for $\Omega^{2}>3 \gamma^{2}$ and $\alpha \Omega<0$; an example is shown in Fig. 1(a). Depending on the initial excitation $\psi_{\mathbf{n}}(0)$ in each decoupled resonator, the dissipative temporal dynamics converges to one of the two stable values of a steady state, $\psi_{\mathbf{n}}(t \rightarrow \infty) \rightarrow \phi_{\downarrow, \uparrow}$.

In the following, we consider the dissipative solitons $\Phi_{\mathbf{n}}$ obtained as the stationary states to which the system converges after sufficient time, $\psi_{\mathbf{n}}(t \rightarrow \infty) \rightarrow \Phi_{\mathbf{n}}$, i.e., in the steady-state limit with $d \psi_{\mathrm{n}} / d t=0$ in Eq. (1). In the first order of the perturbation theory we can approximate the soliton, $\Phi_{\mathbf{n}}=\phi_{\mathbf{n}}\left(1+k \delta_{\mathbf{n}}\right)$, as a binary distribution, $\phi_{\mathbf{n}}=$ $\phi_{\downarrow, \uparrow}$, with the additional corrections $\delta_{\mathbf{n}}$ due to the nonzero coupling $k$. We study spatially extended chains of linked (coupled) sites, for example, a "bright" soliton along a contour $\mathbf{s}, \phi_{\mathbf{s}}=\phi_{\uparrow}$, on the background of low-amplitude stable magnetization, $\phi_{\mathbf{n} \neq \mathbf{s}}=\phi_{\downarrow}$. Such solutions are exact in the anticontinuum limit, as the bistability allows for an arbitrary two-level steady state, $\Phi_{\mathbf{n}}(k=0)=\phi_{\mathbf{n}}$, with $\delta_{\mathbf{n}} \equiv 0$. However, in contrast to the homogeneous solutions with $C_{\mathbf{n}}(\phi) \equiv 0$, the coupling does not vanish, $C_{\mathbf{n}}^{(0)} \equiv C_{\mathbf{n}}\left(\phi_{\mathbf{n}}\right) \neq 0$, and it is a nontrivial question which spatial configurations $\Phi_{\mathbf{n}}$ can be supported with coupled resonators, $k \neq 0$.

Keeping linear terms only, we derive the equations for the corrections, $a_{\mathbf{n}} \delta_{\mathbf{n}}+b_{\mathbf{n}} \delta_{\mathbf{n}}^{*}+C_{\mathbf{n}}^{(0)}=0$, with $b_{\mathbf{n}}=$ $\alpha\left|\phi_{\mathbf{n}}\right|^{2} \phi_{\mathbf{n}}, a_{\mathbf{n}}=b_{\mathbf{n}}-\Sigma$, and the solution

$$
\delta_{\mathbf{n}}=\frac{b_{\mathbf{n}} C_{\mathbf{n}}^{(0) *}-C_{\mathbf{n}}^{(0)} a_{\mathbf{n}}^{*}}{\left|a_{\mathbf{n}}\right|^{2}-\left|b_{\mathbf{n}}\right|^{2}}
$$

Numerical analysis of Eq. (3) shows that the corrections $\delta_{\mathbf{n}}$ remain small in the whole interval of bistability, with the exclusions of small end regions of the $S$ curve in Fig. 1(a). Nevertheless, even for small coupling $k$, the dynamics of the relaxation to the steady-state solution is essentially nonlocal and involves a large number of sites.

The stability of the homogeneous nonlinear states was studied analytically in Ref. [15], and the dynamics of onedimensional discrete switching waves and solitons was explored numerically in Ref. [20]. In general, the stability of a particular configuration depends crucially on its position with respect to boundaries and the coupling strength; here, it is complicated by the fact that the coupling in Eq. (2) is strongly anisotropic. Thus, the stability of each configuration needs to be tested numerically.

To illustrate the features of our system, we begin with the simplest case of a single-site discrete soliton;
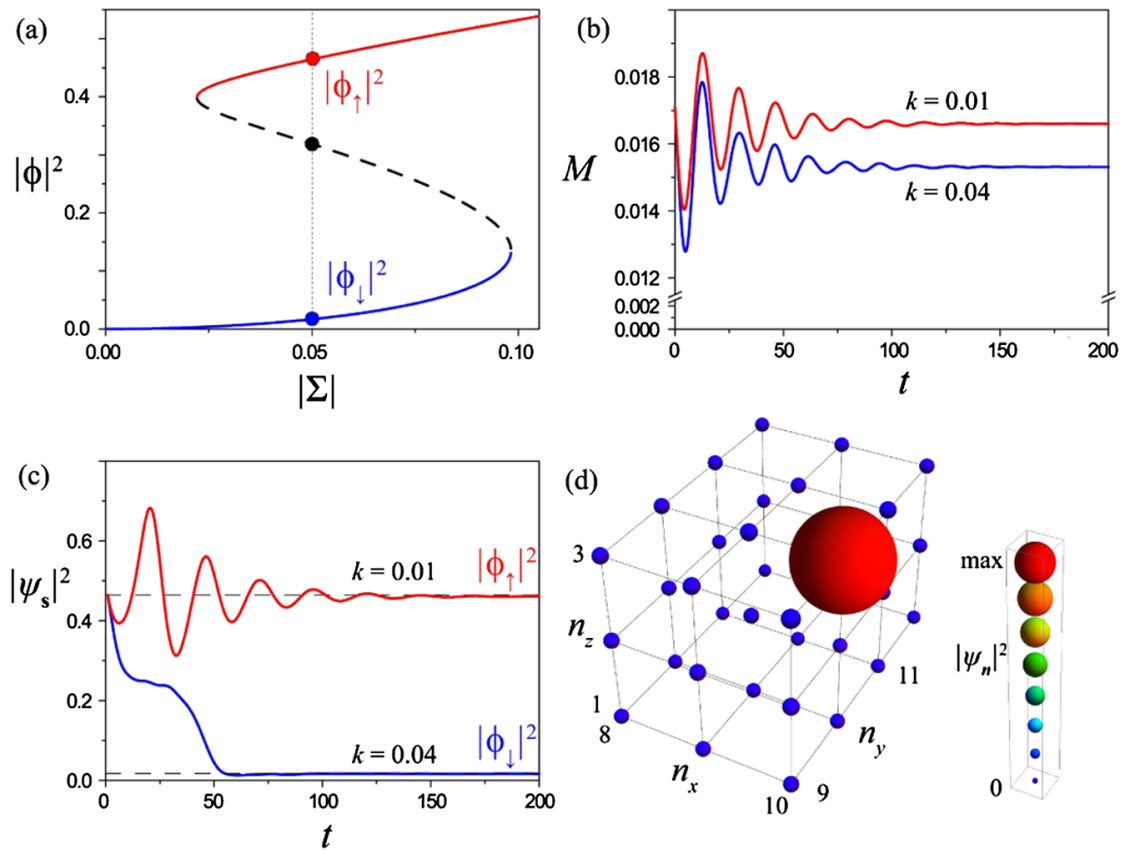

FIG. 1 (color online). (a) The $S$-shaped bistability curve; here and below, $\Omega=-0.2, \gamma=0.035$, and $\alpha=1$ for metallic SRRs of radius $r_{0}=2.5 \mathrm{~mm}$ and inter-resonator spacing of $2.4 r_{0}$ [20]. Temporal dynamics of the (b) total and (c) peak magnetization of a single-site discrete soliton for $\Sigma=0.05$ and two values of the coupling strength $k$ indicated next to the curves. (d) The stable steadystate solution for $\Sigma=0.05$ and $k=0.01$ : the size and color of each sphere is proportional to the magnetization $\left|\psi_{\mathbf{n}}\right|^{2}$, as shown in the inset scale bar. 
the results are shown in Figs. 1(b)-1(d). We choose a large $20 \times 20 \times 5$ slab, to avoid the possible influence of boundaries, with parameters indicated by dots in Fig. 1(a) and one central site initially excited, namely, $\psi_{10,10,3}(0)=\phi_{\uparrow}$, i.e., the soliton profile of just one site, $\mathbf{s}=(10,10,3)$. The dynamics of total magnetization, $M(t)=$ $\sum_{\mathbf{n}}\left|\psi_{\mathbf{n}}\right|^{2} / N_{x} N_{y} N_{z}$, is shown in Fig. 1(b) for two characteristic coupling strengths. Note that, in both cases, the system successfully converges to a steady state. The difference between the two cases is illustrated in Fig. 1(c), with the amplitude of the excited site $\left|\psi_{\mathrm{s}}(t)\right|^{2}$ converging to the upper branch $\phi_{\uparrow}$ for $k=0.01$ and to the background lower branch $\phi_{\downarrow}$ for $k=0.04$; a more detailed numerical study shows that the value $k_{\text {stab }}=0.032$ distinguishes between the two scenarios. The stable soliton is plotted in Fig. 1(d) and shows strong localization practically on a single site. We also found that the influence of the soliton position on its stability threshold is minimal for this choice of parameters, although it is noticeably improved at the boundary, e.g., $k_{\text {stab }}=0.036$ for $s_{z}=1$ or 5 and $k_{\text {stab }}=0.041$ in a single-layer structure $N_{z}=s_{z}=1$.

Next, we search for the linear-segment solitons as building blocks for more extended three-dimensional chains in our cubic lattice. We can introduce the distinction between the stable chain soliton for subcritical coupling, $k<k_{\text {stab }}$, and the unstable (broken) chain for supercritical coupling, $k>k_{\text {stab }}$. For an integral (unbroken) chain of resonators in the upper state, $\phi_{\uparrow}$, the distance between neighbors within the chain is a unit length, and the minimum distance from the chain to other excited resonators (not belonging to the chain) exceeds the unit length. An example for a five-site vertical segment is shown in Figs. 2(a)-2(d). Similar to the single-site soliton above, for small coupling, the chain is stable in Fig. 2(a), while, close to the stability threshold, we obtain a modulated chain-segment soliton in Fig. 2(b), before the steady state shows two decoupled segments in Fig. 2(c). Although, in all these cases, the final states in Figs. 2(a)-2(c) are stable steady-state solutions, the chain in Fig. 2(c) is broken, and thus we determine the chain stability threshold as $k_{\text {stab }} \simeq 0.008$.

The mechanism of instability is illustrated in Fig. 2(d), where the currents between sites, $J_{n}$, are plotted for different values of coupling strength $k$. We find that the currents are negligible in the region of stability, but the modulation grows quickly as $k$ approaches $k_{\text {stab }}$, and the soliton in Fig. 2(b) can be described as a chain of alternating sources and sinks. The current is again negligible for $k>k_{\text {stab }}$ in Fig. 2(c).

We performed a similar stability analysis for vertical and horizontal chain segments of different length $L$; the results are summarized in Fig. 2(e). In general, the stability threshold is higher for horizontal segments at the surface [triangle markers in Fig. 2(e)] than in the bulk (squares), and it is several times higher than that for vertical segments

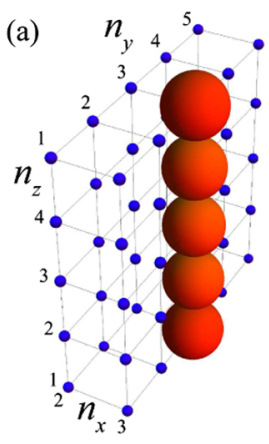

(d)

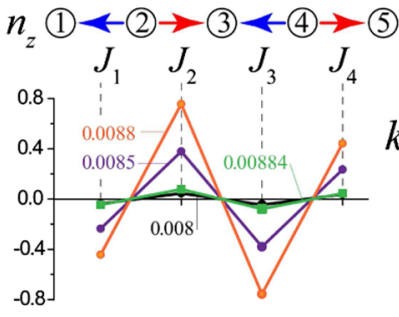

FIG. 2 (color online). Steady-state vertical linear-segment solutions in a $5 \times 5 \times 5$ slab for (a) $k=0.008$, (b) $k=0.0088$, and (c) $k=0.00884$; the scale is as in Fig. 1(d). Corresponding currents, $J_{n}=2 \operatorname{Im}\left[\psi\left(n_{z}\right) \psi^{*}\left(n_{z}-1\right)\right]$, are shown in (d) with numbers indicating $k$ values. (e) The stability diagram for horizontal and vertical linear-segment solitons of length $L$; a soliton is stable for $k<k_{\text {stab }}$ and unstable otherwise.

(circles); the latter is determined for slabs with $5 \times 5 \times L$ sites. The instability of horizontal chains follows the scenario similar to the case of vertical chains-see Fig. 2(d) - and the difference in stability between the two is the consequence of strong coupling anisotropy. The dependence of thresholds on the size of the slab is indicated by large markers in Fig. 2(e).

The results above suggest that the optimal (more robust) lowest-order knot, the unknot, should be built with a horizontal chain, as in Fig. 3(a). In addition to the instabilities of the chain segments, we also observed the instability of the corners of the rectangular chains in Fig. 3(a), such as filling the rectangle with excited sites, as in Fig. 3(b) for $k=0.03$ [21]. The stability thresholds here are lower than that for a horizontal chain itself, such as $k_{\text {stab }}=0.026$ in Figs. 3(a) and 3(b), but always higher than those for vertical segments. The stability border of complex states is determined by the threshold for the weakest link, here the vertical segments. Therefore, we choose lattices with a small number of layers $N_{z}$ which also guarantee applicability of the homogeneous approximation for the pump $\Sigma$.

The initial conditions for different knotted chains in our simulations were chosen in order to maximize the occupancy of the upper state $\phi_{\uparrow}$ at the top, $n_{z}=N_{z}$, and the bottom, $n_{z}=1$, horizontal layers; we also chose simple segments of straight lines. These segments are then linked 


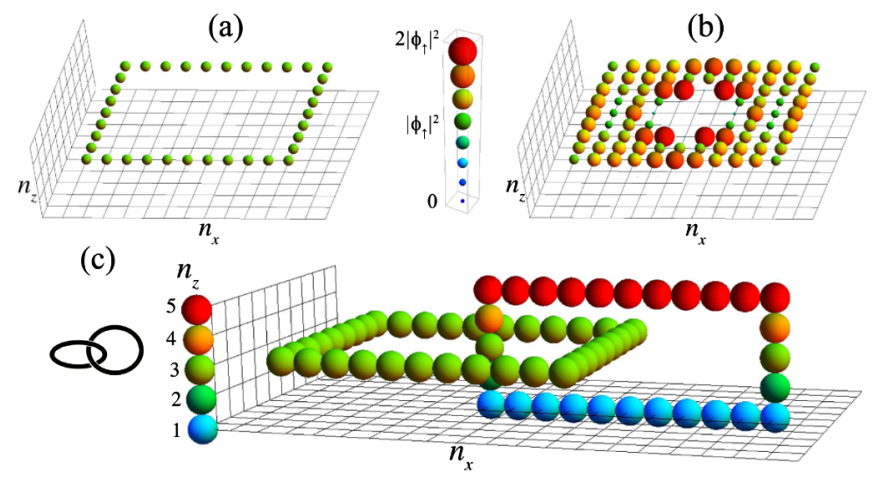

FIG. 3 (color online). Unstable temporal dynamics of the unknot at $t=0$ in (a) and $t=150$ in (b) [21]. (c) Stable Hopf link soliton for $k=0.008$; here and below, in Figs. 4 and 5, the color of spheres encodes the value of $n_{z}$ for better visibility.

by vertical fragments at constant transverse locations $\left(n_{x}, n_{z}\right)$; the number of vertical segments is minimized. This way, knotted lines of arbitrary complexity can be built as initial excitation in a cubic lattice. Naturally, while this is not a unique method to construct knotted lines, it appears to be the most straightforward for our discrete dissipative system.

A knot different from an unknot with two crossings in its projection, as in the inset in Fig. 3(c), is the Hopf link, consisting of two linked unknots. Note that, to link two discrete loops in Fig. 3(c) without crossings in a vertical direction, we need at least $N_{z}=5$ sites, which agrees well with Fig. 2(e), as the stability of the vertical chain does not improve with the increase of corresponding length $L$. Thus, in the following, we keep $N_{z}=5$ and decouple lines in horizontal directions, taking extended states with $N_{x, y}=40$. The stability threshold for the Hopf link coincides with those for the vertical chains, as they break first, with an increase of coupling $k$, as shown in Figs. 2(a)-2(c).

A similar stability threshold and the breakup scenario are found for the trefoil knot shown in Fig. 4. Here, the right column with a sequence of frames, from top to bottom, shows how the vertical segments break up in time, starting with the initial condition in Fig. 4(a) down to the final steady state in Fig. 4(b). We conclude that the trefoil knot in Fig. 4 is unstable for $k=0.01$, although, of course, the stable solitons take the form of Fig. 4(a) for $k<0.008$.

Finally, we illustrate a freedom in building more complex stable knotted solitons in our system with a figure-eight knot and a cinqefoil knot in Fig. 5.

In conclusion, we have revealed the existence of stable knotted solitons in dissipative discrete systems describing nonlinear magnetic metamaterials. We have explored stable and unstable dynamics of knotted solitons and found the stability threshold in terms of the strength of the

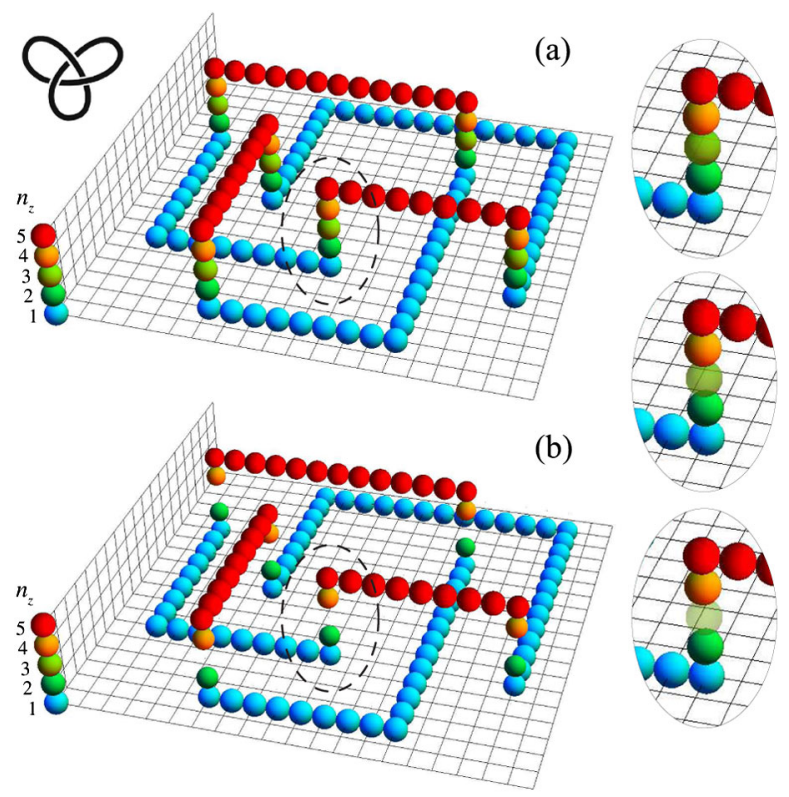

FIG. 4 (color online). Temporal dynamics of an unstable trefoil knot chain for $k=0.01$. The initial knot in (a) breaks in time on its weakest vertical links, as the right column shows, while it converges to the steady state in (b). Here, the local magnetization strength is encoded in a sphere's transparency. Note that (a) also shows a stable trefoil soliton for $k<0.008$.

intersite coupling. These results can be generalized to the lattices of different symmetries with anisotropic coupling, and they will be useful in other fields of physics, chemistry, and biology where the dissipative dynamics and knotted structures are important.

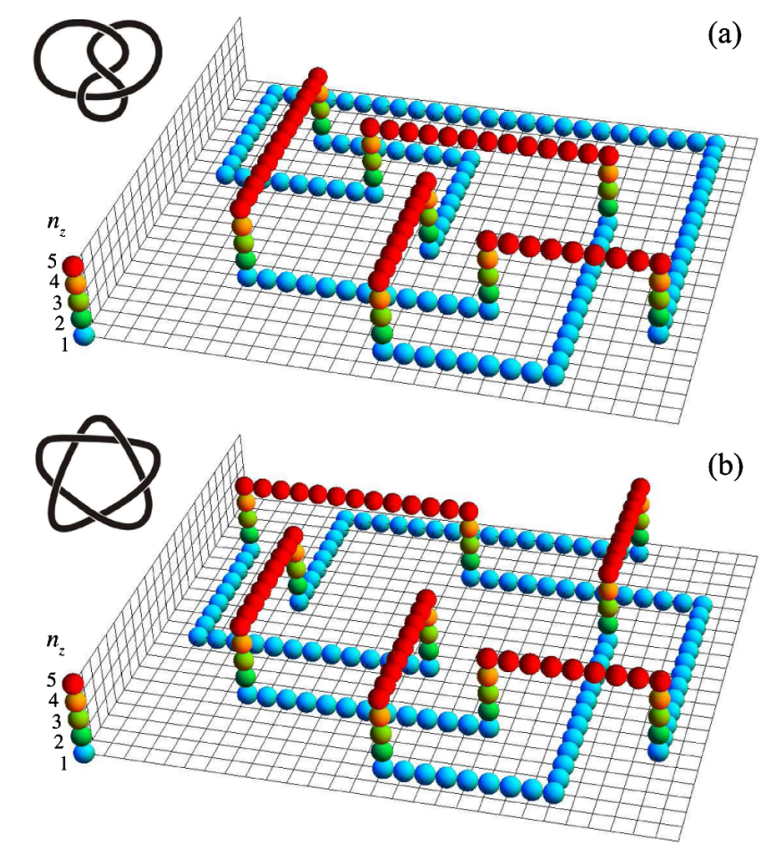

FIG. 5 (color online). Higher-order knotted solitons with 4 and 5 crossings: (a) figure-eight and (b) cinquefoil knots. 
This work was supported by the Australian Research Council, the Ministry of Education and Science of Russia, and ITMO.

[1] L.F. Liu et al., Cell 19, 697 (1980).

[2] J.-F. Ayme et al., Nature Chem. 4, 15 (2011).

[3] F. Y. Wu, Rev. Mod. Phys. 64, 1099 (1992).

[4] I. I. Smalyukh et al., Nature Mater. 9, 139 (2010); U. Tkalec et al., Science 333, 62 (2011).

[5] A. T. Winfree, Nature (London) 371, 233 (1994).

[6] W. H. Thomson (Lord Kelvin), Proc. R. Soc. Edinburgh VI, 94 (1867).

[7] M. V. Berry and M. R. Dennis, Proc. R. Soc. A 457, 2251 (2001).

[8] J. Leach et al., Nature (London) 432, 165 (2004); M. R. Dennis et al., Nature Phys. 6, 118 (2010).

[9] L. D. Faddeev and A. J. Niemi, Nature (London) 387, 58 (1997); Phys. Rev. Lett. 85, 3416 (2000).

[10] J. Gladikowski and M. Hellmund, Phys. Rev. D 56, 5194 (1997); R. A. Battye and P. M. Sutcliffe, Phys. Rev. Lett. 81, 4798 (1998).
[11] E. Babaev, L.D. Faddeev, and A. J. Niemi, Phys. Rev. B 65, 100512(R) (2002); Y. Kawaguchi, M. Nitta, and M. Ueda, Phys. Rev. Lett. 100, 180403 (2008).

[12] E. Babaev, Phys. Rev. Lett. 88, 177002 (2002); J. Garaud, J. Carlström, and E. Babaev, ibid. 107, 197001 (2011).

[13] N. N. Rosanov, Spatial Hysteresis and Optical Patterns (Springer, Heidelberg, 2002).

[14] A. A. Zharov, I. V. Shadrivov, and Yu. S. Kivshar, Phys. Rev. Lett. 91, 037401 (2003).

[15] I. V. Shadrivov et al., Photon. Nanostr. Fundam. Appl. 4, 69 (2006).

[16] N. Lazarides, M. Eleftheriou, and G.P. Tsironis, Phys. Rev. Lett. 97, 157406 (2006).

[17] Nonlinearities in Periodic Structures and Metamaterials, edited by C. Denz, S. Flach, and Yu. S. Kivshar (Springer, Heidelberg, 2010).

[18] I. V. Shadrivov et al., Opt. Express 16, 20266 (2008).

[19] D. A. Powell et al., Phys. Rev. B 82, 155128 (2010).

[20] N. N. Rosanov et al., JETP Lett. 93, 743 (2011); Opt. Express 19, 26500 (2011).

[21] See Supplemental Material at http://link.aps.org/ supplemental/10.1103/PhysRevLett.108.133902 for the development of instability of the unknot in Figs. 3(a) and 3(b). 